\title{
Исследование процесса деионизации растворов электросорбцией на аэрогельных электродах посредством математического моделирования
}

\author{
Тихонов Н.А., Токмачев М.Г. \\ Московский государственньй университет им. М.В. Ломоносова, Москва
}

Поступила в редакцию 25.11.2017 г.

\begin{abstract}
В работе изучается динамика деионизации раствора электролита с помощью сорбции на аэрогельных электродах на основе математического моделирования. Учитываются процессы переноса вещества потоком раствора, диффузии и сорбции в порах. Предложены модели, описывающие явление с разной степенью подробности. Показаны трудности, возникающие при численных расчетах процесса, и возможности их преодоления.

Показано, что при низких концентрациях раствора и малом размере пор эффект электросорбции не сводится к образованию на поверхности пор двойного электрического слоя, отбирающего ионы из раствора. Кроме формирования этого слоя, происходит накопление распределенного заряда ионов во всем объеме пор. Приведены примеры расчета процесса деионизации в циклическом режиме.
\end{abstract}

Ключевые слова: деионизация, аэрогельные электроды, математическое моделирование.

\section{Investigation of the process of solution deionization by electro-sorption on aerogel electrodes via mathematical modeling}

\author{
Tikhonov N.A., Tokmachev M.G. \\ Moscow Lomonosov State University, Moscow
}

Dynamics of deionization of electrolyte solution by sorption on aerogel electrodes is studied in the work on the basis of mathematical modeling. The processes of substance transfer by solution flow, diffusion and sorption in pores are taken into account. The models describing the phenomenon with varying degrees of detail are proposed. Difficulties encountered at numerical calculations of the process and the possibilities of their overcoming are shown.

It is demonstrated that at low solution concentrations and a small pore size the electro-sorption effect does not reduce to the formation of an electrical double layer on the pore surface that takes ions out of solution. In addition to the formation of the layer, there is an accumulation of a distributed ion charge in the entire volume of the pores. Calculation examples of the deionization process in a cyclic regime are given.

The influence of the different physical parameters such as the concentration of the electrolyte, the size and shape of the aerogel electrode pores, solution flow rate, length and thickness of the channel between electrodes, thickness of the electrodes and their electrical conductivity is studied on the of the output curve shape and the performance of the process. Some analytical assessments of the purified solution volume at various deionization degrees have been got.

Keywords: deionization, aerogel electrodes, mathematical modeling. 


\section{Введение}

Метод емкостной деионизации (МЕД) водных растворов - это новый перспективный и наиболее экономичный метод опреснения воды, который заключается в прокачке водного раствора через электрохимическую ячейку между двумя пористыми электродами с высокоразвитой поверхностью (такие как углеродные аэрогели), между которыми задается определенная разность потенциалов $\sim 1.2$ В. В качестве электродов обычно используются углеродные электроды с высокой удельной поверхностью $\mathrm{S}=500-2500 \mathrm{~m}^{2} / \Gamma$, на которых происходит адсорбция ионов и как следствие - деионизация воды [1].

Работа установки, основанной на МЕД, обычно проходит через две фазы: фазу адсорбции, где вода опресняется, и фазу десорбции, где электроды регенерируются. Во время фазы адсорбции накладывается разность потенциалов на два электрода, и ионы адсорбируются из воды. После того, как электроды насыщаются ионами, полярность электродов меняют местами или разность потенциалов уменьшают до нуля. Таким образом, ионы покидают поры электрода и могут быть вымыты из ячейки, что приводит к выходу потока с высокой концентрацией соли, так называемого потока рассола или концентрата. При этом, часть энергии, требуемой во время фазы адсорбции, может быть восстановлена на этапе десорбции [2-3].

В настоящее время МЕД в основном используется для опреснения солоноватой воды, которая представляет собой воду с низкой или умеренной концентрацией соли (ниже 10 г/дм³) [4-6]. Другими технологиями деионизации воды являются, в частности, дистилляция, обратный осмос и электродиализ. По сравнению с обратным осмосом и дистилляцией, МЕД считается энергоэффективной технологией опреснения солоноватой воды [5]. Это происходит главным образом потому, что в этом методе удаляются ионы соли из воды, в то время как другие технологии экстрагируют воду из раствора соли.

Имеется много различных экспериментальных исследований МЕД, их литературный обзор можно найти, например, в [1, 4, 6-9]. В основном исследования направлены на изучение равновесия между концентрациями различных электролитов во внешней области аэрогелей и сорбцией их внутри аэрогеля, энергозатраты, а также возникающие в переменных режимах токи, характеризующие динамику деионизации [8-11].

Качественное объяснение механизма процесса в порах аэрогеля в первом приближении может быть описана с помощью модели Гуи-Чепмена-Штерна, согласно которой вблизи поверхности образуются три разных слоя: пористая матрица аэрогеля, которая содержит электрический заряд в своей структуре, слой Штерна и диффузный слой [12]. Для формирования такой структуры происходит отбор ионов из раствора.

Во многих работах теоретический подход по существу сводится к представлению аэрогелных электродов, как поверхности конденсатора большой емкости [11, 13-17] собирающего ионы из промежутка между электродами, по которому происходит движение раствора. Соответственно, для описания процесса при этом используются так называемая одномерная модель пористых электродов. Таким способом обоснованно рассматривать и рассчитывать процесс деионизации, в том числе в циклическом режиме, если поры аэрогеля достаточно крупные и промежуток между электродами, по которому происходит перенос раствора, относительно широкий. В этом случае лимитирующей стадией процесса является перенос ионов внутри промежутка к поверхности электродов, а более тонкие явления внутри аэрогеля не существенны. В то же время, если поставить задачу определения параметров, при ко- 
торых процесс деионизации наиболее эффективен, то ситуация меняется. Диаметр пор следует уменьшать. Применение материалов, содержащих поры в виде нанотрубок, показало высокую способность к накоплению заряда [18]. Может меняться отношение толщины промежутка между электродами к толщине электродов и увеличиваться скорость пропускания раствора по промежутку. В этом случае лимитирующей стадией процесса деионизации будет являться диффузия ионов внутри электрода. Кроме того, строение любого аэрогеля неоднородно. Для определения оптимальных характеристик деионизатора необходимы модели, которые обеспечивают учет пространственного распределения заряда внутри электродов. Целью математического моделирования является не только описание имеющихся экспериментальных данных, но и предсказание процесса деионизации во всех подробностях. Модель, где рассматривается перенос ионов не только по промежутку между электродами, но и внутри пористого аэрогеля, предложена в [19]. Это двумерная модель переноса вещества. В целом, она базируется на фундаментальных законах физики, однако ее слабым местом является описание связи заряда и концентрации ионов в поpe.

При фиксированной порозности аэрогеля чем меньше диаметр пор, тем больше их суммарная поверхность и тем эффективнее протекает деионизация раствора. Однако процесс, происходящий в тонких порах, существенно осложнен лимитирующим влиянием диффузии ионов, влиянием на скорость диффузии сильно неоднородного электрического поля, зависимости мощности двойного электрического слоя от радиуса пор и их формы. Поэтому, мы думаем, что требуется дальнейшее, более подробное теоретическое исследование процесса деионизации, проводимого в циклическом режиме, с учетом указанных эффектов. Такие исследования возможны на основе математического моделирования, которое способно давать количественный результат для переменного циклического процесса.

\section{Физическая модель}

Пусть имеется плоский конденсатор (рис. 1), состоящий из металлических пластин (a) длины $L z$. На пластины нанесены слои (b) толщины $H$ электропроводящего пористого вещества. Будем называть его сорбентом. Между слоями расстояние $L$. В дальнейшем пространство между слоями сорбента будем называть «каналом».

Будем считать $L z$ порядка нескольких см, $L-$ нескольких мм, $H$ - долей мм. Порозность сорбента обозначим как $\eta$. По каналу происходит перенос электролита. $\mathrm{B}$ начальный момент сорбент заполнен электролитом концентрации $C_{0}$. Такая же концентрация поддерживается на входе в канал $(z=0)$. Будем считать $C_{0}$ величиной порядка долей моль/литр. Далее везде мы будем концентрацию указывать в моль/дм ${ }^{3}$. Будем считать, что физические характеристики ионов разного знака, в том числе их коэффициенты диффузии, одинаковы. Тогда, очевидно, в любой момент времени распределение концентраций ионов симметрично относительно середины канала, и мы можем рассматривать происходящее только в одном слое сорбента.

В модельном рассмотрении изучим два случая: будем поры представлять в виде тонких цилиндров радиуса $R_{0}$ и в виде тонких щелей ширины $2 R_{0}$ глубины $H$, расположенных перпендикулярно пластинам, а скелет сорбента - в виде пространства между порами, затемненного на рис. 2. Ориентируясь на данные из работ $[5,20]$, считаем $R_{0} \approx 10$ нм.

Пусть в начальный момент, при $t=0$, разность потенциалов между обкладками конденсатора равна 0 , а затем на время $0<t<T$ ключ замыкается и разность 
потенциалов равна $\Phi$. Считаем $\Phi \approx 1.2$ Вольта - меньше напряжения, при котором начинается гидролиз воды.

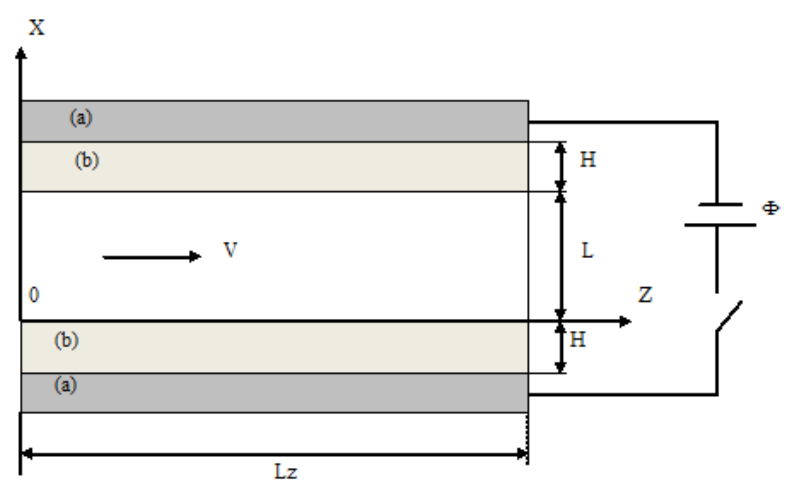

Рис.1. Схема деионизатора

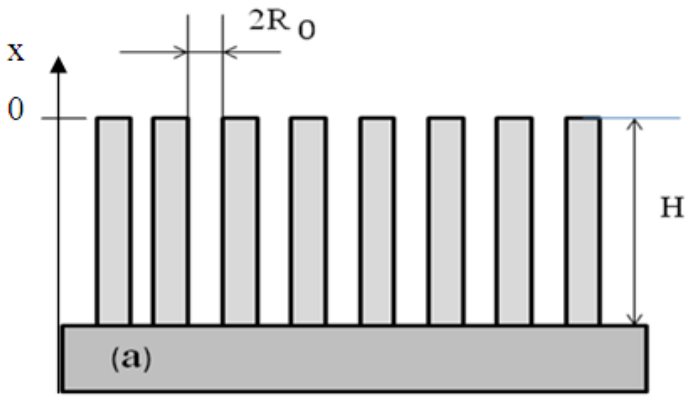

Рис. 2. Схема модели пористого электрода

После подачи напряжения ионы притягиваются к заряженной поверхности пор. Вблизи поверхности накапливается вещество в виде гидратированных ионов и происходит деионизация раствора в канале. Наша задача состоит в том, чтобы составить модель, допускающую расчет процесса, и построить зависимость количества собранного у поверхности вещества и концентрацию выходящего из канала раствора от времени при различных параметрах физической модели.

\section{Математическая модель}

Пусть $C_{ \pm}, j_{ \pm}$обозначают локальные концентрации ионов и плотность потоков ионов соответствующего знака. Обозначим концентрацию электролита в канале как $C$, локальное значение потенциала в растворе, отсчитанного от середины канала - как $\varphi$.

Вначале рассмотрим случай, когда электропроводимость сорбента достаточно высокая, а толщина слоя $H$ мала по сравнению с $L$. При этом время перераспределения зарядов по скелету сорбента много меньше характерного времени диффузии и переноса раствора по каналу. Поэтому считаем потенциал во всех точках скелета сорбента равным потенциалу пластины конденсатора, на которую он нанесен.

При равновесии значения $C_{ \pm}$согласно закону распределения Больцмана равны $C_{ \pm}=C e^{\mp u}$, где $u=\frac{\varphi q}{k T}$. Здесь $q$ - заряд электрона, $k$ - постоянная Больцмана, $T$ - температура. Поэтому удобно рассматривать нормированный потенциал $u$. Обозначим $\frac{\Phi q}{2 k T}$ как $u_{0}\left(u_{0} \approx 23\right)$.

Получаем систему уравнений описывающих процесс:

$$
\begin{gathered}
\frac{\partial C_{ \pm}}{\partial t}+v \frac{\partial C_{ \pm}}{\partial z}=-d i v j_{ \pm}-\text {уравнение переноса в области } 0<x<L . \\
j_{ \pm}=-D\left(\nabla C_{ \pm} \pm C_{ \pm} \nabla u\right) \text { - уравнение Нерста - Планка. } \\
\Delta u=\mu\left(C_{-}-C_{+}\right) \theta \text { - уравнение Пуассона. }
\end{gathered}
$$

Здесь $v$ - скорость потока раствора в канале, $D$ - коэффициент диффузии, $t-$

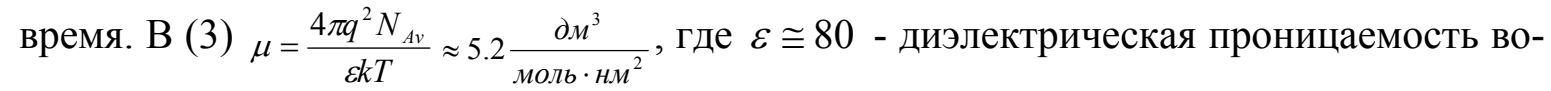
ды, а $N_{A v}$ - число Авогадро. Гидратированный ион не может быть расположен на 
расстоянии, меньшем радиуса гидратной оболочки $r_{\text {гид }}$ от поверхности сорбента. Отсюда, в (3) появляется коэффициент $\theta$, причем $\theta=0$ в точках вблизи поверхности, недоступных для ионов, и $\theta=1$ в остальном пространстве. Значение $r_{\text {zuд }}<<R_{0}$. Однако, $e^{u}$ очень велико вблизи поверхности. Потому величиной $r_{\text {гuд }}$ при расчете интегралов нельзя пренебрегать. В дальнейшем $R_{0}-r_{\text {гид }}$ обозначим как $R_{1}$.

Систему (1)-(3) нужно дополнить начальными и граничными условиями:

$\left.\frac{\partial u}{\partial z}\right|_{z=0}=\left.\frac{\partial u}{\partial z}\right|_{z=L z}=0$ - пренебрегаем искривлением силовых линий на краях конденсатора.

$\left.C_{ \pm}\right|_{z=0}=C_{0}$ - условия на входе в канал.

$\left.\frac{\partial C_{ \pm}}{\partial z}\right|_{z=L z}=0$ - условие свободного выхода раствора из канала.

$\left.C_{ \pm}\right|_{t=0}=C_{0}$ - начальные условия.

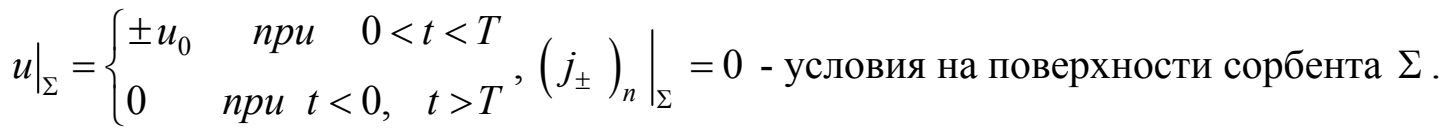

В последних соотношениях $\Sigma$ - это вся поверхность сорбента, в том числе поверхность скелета, ограничивающая поры внутри слоя сорбента; знак \pm перед $u_{0}$ выбирается в соответствии со знаком заряда слоя сорбента; $j_{n}$ - нормальная составляющая потока. Приведенные соотношения образуют полную математическую модель рассматриваемого процесса. Обозначим их, как модель (I).

\section{Модификация модели (I)}

Модель имеет достаточно простой вид, но численный расчет с ее помощью связан со значительными трудностями. Для удобства иллюстраций будем рассматривать слой сорбента, примыкающий к обкладке конденсатора, на которой $\mathrm{u}_{0}>0$. (Нижний слой на рис.1) Для состояния равновесия рассчитаем распределение потенциала $u$ в некотором сечении цилиндрической поры, перпендикулярном направлению $x$. Из (3) с учетом закона распределения Больцмана получаем $\Delta u=\mu C\left(e^{u}-e^{-u}\right) \theta$. Пусть $C \cong 10^{-5}-10^{-1}$ моль/дм ${ }^{3}$. Тогда величина $\mu C$ - велика и распределение $u(r)$, где $r$ расстояние от продольной оси поры до точки наблюдения, имеет рассчитанный нами вид, приведенный на рис.3.

В узкой области вблизи стенки поры (на рисунке справа), на расстоянии порядка десятка ангстрем, значение $u$ резко изменяется. В этой области значение выражения $C\left(e^{u}-e^{-u}\right)$ весьма велико, следовательно, здесь собран заряд в виде ионов знака противоположного заряду сорбента. Этот заряд блокирует поле, поэтому в остальной части сечения поле относительно невелико. При этом средняя концентрация по сечению поры существенно больше равновесной концентрация в канале $C$. Имеет место накопление целевого компонента в порах - явление, по внешнему виду похожее на сорбцию вещества.

Обратим внимание на то, что при малых значениях $C$ «затягивание» ионов из канала происходит не только непосредственно к стенкам пор, но и в некоторую более широкую область. Например, на верхней кривой на рис. 3 в центре поры значе- 
ние $u \approx 6$. Это означает, что в центре поры концентрация ионов примерно в $e^{6} \approx 400$ раз больше, чем в канале. В этом случае недостаточно ограничиться представлением о процессе, как о формировании тонкого двойного слоя у поверхности пор.

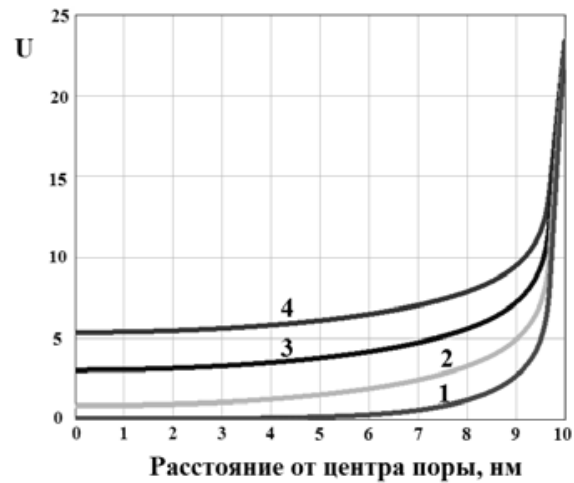

Рис. 3. Распределение потенциала по сечению поры.

Кривая 1 соответствует $C_{1}=0.1$, кривая $2-C_{2}=0.01$, кривая $3-C_{3}=0.001$, кривая $4-C_{4}=0.0001$.

Пусть сечение поры имеет площадь $S$. Выберем центральную часть сечения поры с площадью $0.9: S$. Тогда, при $R_{0}=10$ нм и $C=10^{-5}$, по нашим расчетам, примерно треть ионов находятся в указанной центральной части. Если аэрогель - микропористый и имеет поры ширины около 2 нм [21], то этот эффект будет еще больше. В этом случае в центральной части поры концентрация ионов будет примерно в 3000 раз больше, чем в канале для $C=10^{-3}$.

Для описания процесса диффузии удобно ввести понятие средней концентрации электролита $\bar{C}$ в некотором сечении поры. Здесь $\bar{C}$ следует понимать как $\bar{C}=\left(\bar{C}_{+}+\bar{C}_{-}\right) / 2$, где $\bar{C}_{ \pm}$средние значения концентраций ионов.

Как уже было отмечено выше, при равновесии в соответствии с распределением Больцмана локальные концентрации ионов в этом сечении равны $C_{ \pm}=C \cdot e^{\mp u}$. Отсюда $\bar{C}=\frac{\bar{C}_{+}+\bar{C}_{-}}{2}=C \cdot \int_{0}^{R_{1}} \frac{e^{u}+e^{-u}}{R_{1}^{2}} r d r$. Обозначим последний интеграл как $I$. Тогда $C=\frac{\bar{C}}{I}$ И

$$
C_{ \pm}=\frac{\bar{C}}{I} e^{\mp u}
$$

Теперь обратимся к трудностям при расчетах с помощью модели (I). Поскольку $\left(e^{u}-e^{-u}\right)$ вблизи границы сорбента порядка $e^{20}$, то для расчета требуется применение сетки, сгущающейся по $r$ на несколько порядков в этом месте.

Кроме того, в порах нет электронейтральности раствора. Поэтому необходимо вычислять потоки как положительных, так и отрицательных ионов, меняющихся по времени в условиях большого градиента концентрации и потенциала. Поскольку необходимо иметь примерно $10^{2}$ шагов по $z$ и того же порядка по $x$, то получается, что шагов разностной сетки нужно порядка $10^{8}$, что нереально. Поэтому нужно не прямо использовать модель (I), а пренебречь некоторыми второстепенными эффектами, и получить модель, в которой расчеты поля в порах выделены в виде отдельного блока, а диффузия в порах описывается соотношениями с одной пространственной переменной х, что существенно упрощает ситуацию.

А именно, пренебрегаем:

- диффузией вдоль канала по сравнению с переносом вещества потоком; 
- градиентом поля вдоль поры по сравнению с градиентом в направлении $r$. Это имеет место, когда длина поры много больше ее ширины. У нас примерно в $10^{4}$ раз;

- в момент подачи напряжения $\left.u\right|_{\Sigma}<0$ на электрод распределение ионов имеет вид, отличный от (4). Ионов $C_{+}$слишком мало, а $C_{-}$слишком много для равновесия. На другом электроде ситуация обратная. Возникают временные потоки ионов разной направленности, компенсирующие друг друга в канале и приводящие распределение $C_{ \pm}$к виду (4). Пренебрегаем этими потоками при описании процесса.

В силу сделанных предположений из (3) и (4) получаем отдельную задачу для расчета $u$ в сечении поры при заданном значении $\bar{C}$. Имеем:

$$
\frac{1}{r} \frac{\partial}{\partial r}\left(r \frac{\partial u}{\partial r}\right)=\mu \bar{C} \theta \frac{\left(e^{u}-e^{-u}\right)}{R_{1}^{-2} \int_{0}^{R_{0}}\left(e^{u}+e^{-u}\right) r d r}, \quad u_{\mid r=R_{0}}=u_{0},\left.\quad \frac{\partial u}{\partial r}\right|_{r=0}=0
$$

Здесь $\theta=0$ при $R_{1}<r<R_{0}$ и $\theta=1$ в остальной части сечения. Из (5) видно, что $I=I(\bar{C})$. Из (5) рассчитываем зависимости $I(\bar{C})$ и $\frac{d I(\bar{C})}{d \bar{C}}$ для разных значений $\bar{C}$.

Плотность диффузионного потока вдоль поры описывается законом НерстаПланка:

$j_{ \pm}=-D\left(\frac{\partial C_{ \pm}}{\partial x} \pm C_{ \pm} \frac{\partial u}{\partial x}\right)$. Запишем это уравнение в виде:

$$
j_{ \pm}=-D \frac{\partial\left(e^{ \pm u} \cdot C_{ \pm}\right)}{e^{ \pm u}}
$$

Из (4) и (6) следует: $j_{ \pm}=-D \frac{\partial}{\partial x}\left(\frac{\bar{C}}{I(\bar{C})}\right) e^{\mp u}$.

Общий поток целевого компонента $J$ через сечение поры будет:

$$
\begin{gathered}
J=2 \pi \int_{0}^{R_{1}} \frac{j_{+}+j_{-}}{2} r d r=-\pi D \int_{0}^{R_{1}} \frac{\partial}{\partial x}\left(\frac{\bar{C}}{I(\bar{C})}\right) \cdot\left(e^{u}+e^{-u}\right) r d r= \\
=-\pi D \int_{0}^{R_{1}}\left(\frac{\frac{\partial \bar{C}}{\partial x} \cdot\left(e^{u}+e^{-u}\right)}{I(\bar{C})}-\bar{C} \frac{d I(\bar{C})}{d \bar{C}} \cdot \frac{\partial \bar{C}}{\partial x} \cdot \frac{e^{u}+e^{-u}}{I(\bar{C})^{2}}\right) r d r=-\pi R_{1}^{2} D \frac{\partial \bar{C}}{\partial x}\left(1-\frac{\bar{C}}{I(\bar{C})} \cdot \frac{d I(\bar{C})}{d \bar{C}}\right)
\end{gathered}
$$

Таким образом, находим величину потока в зависимости от концентрации $\bar{C}$ и ее производной $\frac{\partial \bar{C}}{\partial x}$.

Заметим, что $\frac{\partial \bar{C}}{\partial x}\left(1-\frac{\bar{C}}{I(\bar{C})} \frac{d I(\bar{C})}{d \bar{C}}\right)=\frac{\partial \bar{C}}{\partial x}-\bar{C} \frac{\partial}{\partial x} \ln I(\bar{C})$. Если $\left.u\right|_{\Sigma}=0$, то $I(\bar{C})=1$ и $J=-D \pi R_{0}^{2} \frac{\partial \bar{C}}{\partial x}$

Обратимся к ситуации на границе $x=0$ канал-сорбент. Поле в канале, с учетом сделанных предположений, удовлетворяет условиям:

$$
\frac{\partial^{2} u}{\partial x^{2}}=\mu C\left(e^{u}-e^{-u}\right),\left.u\right|_{x=0}=u_{0}, u_{\mid x=L}=-u_{0}
$$

Решение этой задачи убывает на участке $0<x<l=\frac{1}{\sqrt{2 \mu C}}$ примерно в 15 раз, а далее $u \approx 4 e^{-x / l} . l$ - величина порядка 10 нм. Средний размер пор $2 R_{0}$ может быть 
больше, но имеет тот же порядок величины. Выберем $x_{1}=1$ мкм. Тогда $\left.u\right|_{x=x_{1}} \approx 0$, a $\left.C\right|_{x=x_{1}}$ не зависит от детального расположения входов в поры из канала. Характерный масштаб изменения концентрации в направлении $x$ вследствие диффузии, как в канале, так и в порах, на несколько порядков превосходит $x_{1}$. Поэтому, при описании процесса диффузии можем считать $\left.C_{ \pm}\right|_{x=x_{1}}$ и $\left.C_{ \pm}\right|_{x=-x_{1}}$ равновесными. Тогда, в соответствии с законом распределения Больцмана локальная концентрация $\left.C_{ \pm}\right|_{x=-x_{1}}=\left.e^{\mp u} C_{ \pm}\right|_{x=x_{1}}$, где $u$ - значение потенциала в рассматриваемой точке. Вычисляя среднее значение концентрации по сечению поры $x=-x_{1}$, находим $\left.C\right|_{x=x_{1}}=\left.\bar{C}\right|_{x=-x_{1}} / I\left(\left.\bar{C}\right|_{x=-x_{1}}\right)$.

Приходим к следующим соотношениям:

$$
\begin{aligned}
& \frac{\partial C}{\partial t}+v \frac{\partial C}{\partial z}=D_{e x} \frac{\partial^{2} C}{\partial x^{2}}-\text { при } x_{1}<x<L / 2 . \\
& \frac{\partial \bar{C}}{\partial t}=D_{i n} \frac{\partial^{2} \bar{C}}{\partial x^{2}} \cdot F(\bar{C}) \text {, где } F(\bar{C})=1-\frac{\bar{C}}{I(\bar{C})} \frac{\partial I(\bar{C})}{\partial \bar{C}}-\text { при }-H<x<x_{1} \text {. } \\
& D_{e x} \frac{\partial C(0, z, t)}{\partial x}=D_{i n} \eta \frac{\partial \bar{C}(0, z, t)}{\partial x} F(\bar{C}(0, z, t)) \\
& C(0, z, t)=\frac{\bar{C}(0, z, t)}{I(\bar{C}(0, z, t))}
\end{aligned}
$$

Здесь $D_{e x}$ и $D_{i n}$ - коэффициент диффузии в канале и в сорбенте.

Зависимость $I(\bar{C})=R_{1}^{-2} \int_{0}^{R_{1}}\left(e^{u}+e^{-u}\right) r d r$ определяется из задачи (5). Соотношения (5), (8)-(12) образуют полную модель, которую будем называть моделью (II).

Модель (II) удобна для расчетов. Все сложности с переменным шагом вычислений относятся только к задаче (5), которая решается отдельно. Таким образом исключается функция $u$, поэтому получается задача диффузии для $C$ и $\bar{C}$ в смежных областях с условиями (10) и (11), связывающими эти функции на границе между областями.

\section{Результаты моделирования}

Обратимся к моделированию концентрации на выходе из канала $\left.C\right|_{z=L z}$ при включении и выключении напряжения на конденсаторе. На рис.4-9 приведены выходные кривые $C_{\text {out }}(t)=\frac{1}{L} \int_{0}^{L} C(L z, x, t) d x$ для разных значений параметров. Расчет велся по модели (II). Если не сказано иного, то по умолчанию использовались следующие значения параметров: порозность $\eta=0.9$; концентрация исходного раствора $C_{0}=0.01$ моль/л; радиус пор $R_{0}=9$ нм; линейная скорость пропускания раствора по каналу $v=0.03 \mathrm{mм} / \mathrm{c}$; толщина слоя сорбента $H=0.2 \mathrm{Mm}$; ширина канала $L=10 \mathrm{mм}$; коэффициент диффузии в канале $D_{e x}=2.410^{-9} \mathrm{~m}^{2} / \mathrm{c}$; коэффициент диффузии в порах $D_{i n}=0.810^{-9} \mathrm{M}^{2} / \mathrm{c}$; время сорбции $T_{\text {sorp }}=40$ мин; время регенерации $T_{\text {reg }}=80$ мин. 
На рис. 4 показано влияние формы пор, а на рис. 5 - влияние размера пор на выходную кривую.

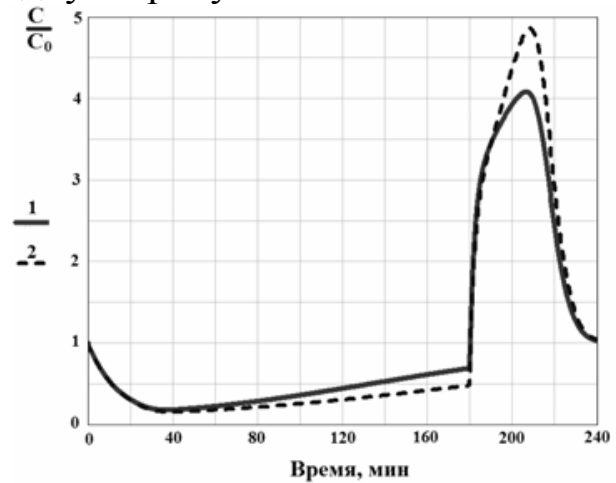

Рис. 4. Иллюстрация влияния формы пор на выходную кривую.

Расчет велся для параметров: $C_{0}=0.001$ моль/дм ${ }^{3} ; T_{\text {sorp }}=180$ мин; $T_{r e g}=60$ мин; Кривая 1 - щелевидные поры; Кривая 2 - цилиндрические поры

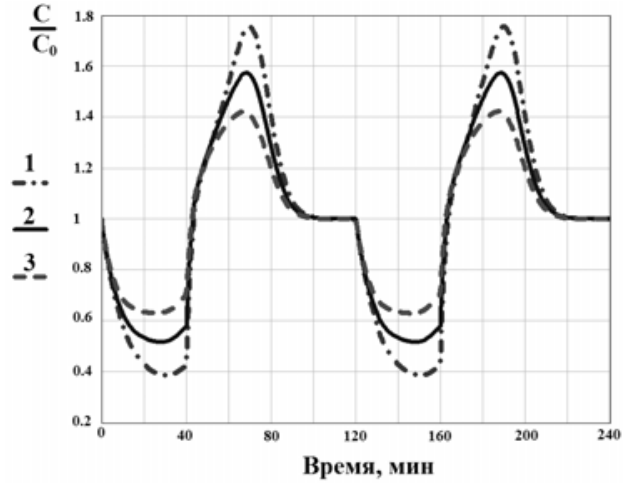

Рис. 5. Иллюстрация влияния размера щелевидных пор на выходную кривую. Кривая $1-R_{0}=9$ нм, кривая $2-R_{0}=20$ нм, кривая $3-R_{0}=40$ нм.

Как видно из представленного графика, уменьшение среднего размера пор улучшает эффективность процесса. На рис. 6 и рис. 7 представлены выходные кривые, полученные для разных концентраций исходного раствора. Можно видеть, что процесс будет тем эффективнее, чем меньше концентрация раствора.

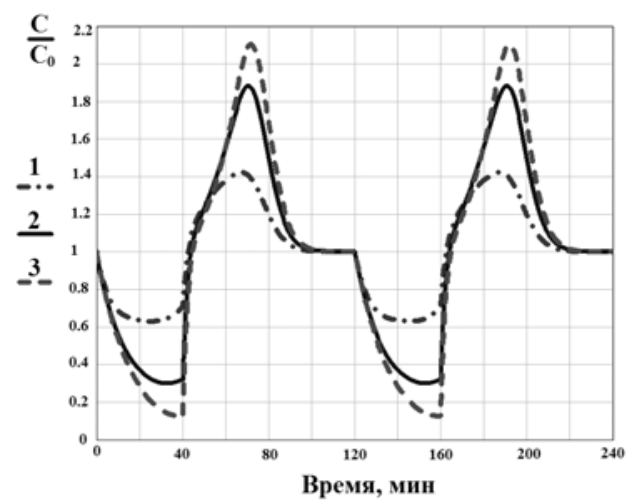

Рис. 6. Иллюстрация влияния концентрации исходного раствора на выходную кривую.

Кривая 1 получена для концентрации исходного раствора $C_{0}=10^{-2}$ моль/дм ${ }^{3}$, кривая $2-$ для $C_{0}=10^{-3}$ моль/дм ${ }^{3}$, кривая 3 - для $C_{0}=10^{-4}$ моль/дм ${ }^{3}$. Расчет велся для щелевидных пор размером $R_{0}=40 \mathrm{Hм}$

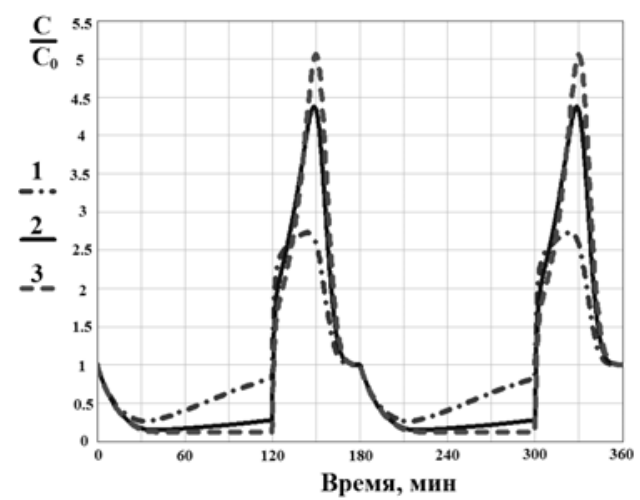

Рис. 7. Иллюстрация влияния концентрации исходного раствора на выходную кривую.

Расчет велся для щелевидных пор размером в слое сорбента толщиной $H=0.1$ мм; длительность сорбции $T_{\text {sorp }}=120$ мин, длительность регенерации $T_{\text {reg }}=60$ мин. Кривая 1 получена для концентрации исходного раствора $C_{0}=10^{-3}$ моль/дм ${ }^{3}$, кривая 2 - для $C_{0}=10^{-4}$ моль/дм ${ }^{3}$, кривая $3-$ для $C_{0}=10^{-5}$ моль/дм ${ }^{3}$.

На рис. 8 показаны выходные кривые для разной толщины канала и скорости пропускания раствора, при этом поток раствора оставался постоянным. Как легко видеть, процесс будет тем эффективнее, чем тоньше канал.

На рис. 9 представлены выходные кривые для разных толщин слоя сорбента аэрогеля на электродах. Как можно видеть из рисунка, глубокие поры (H 1 мм) способствуют поглощению большего количества вещества на этапе сорбции, однако дают более плавные кривые на этапе регенерации. Неглубокие поры (H 0.1 мм) бы- 
стрее заполняются на этапе сорбции и дают более резкое высвобождение вещества на этапе регенерации. Поэтому этот параметр нужно подбирать аккуратно, исходя из конкретных экспериментальных задач.

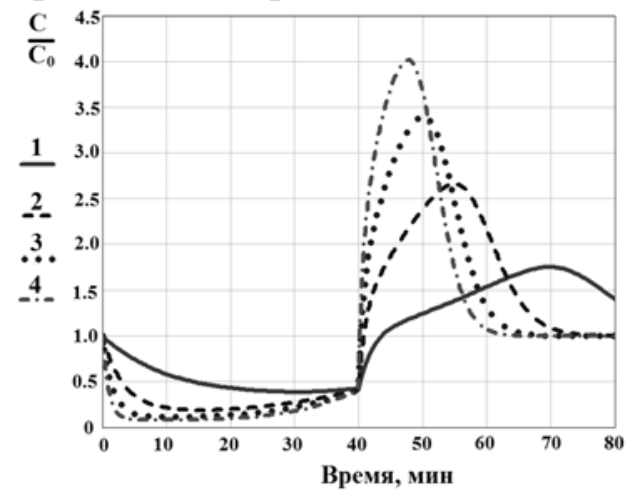

Рис. 8. Иллюстрация влияния скорости движения раствора на выходную кривую при фиксированной величине потока.

Расчет велся для $T_{\text {sorp }}=T_{\text {reg }}=40$ мин. Кривая 1 : $v=0.03 \mathrm{мм} /$ сек; $L=10$ мм; Кривая $2: v=0.06 \mathrm{mм} /$ сек;

$L=5$ мм; Кривая 3: $v=0.09$ мм/сек; $L=3.33$ мм; Кривая 4: $v=0.12$ мм/сек; $L=2.5$ мм

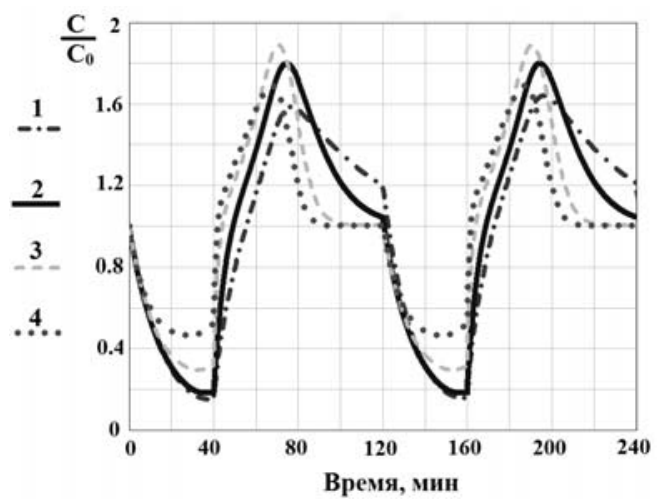

Рис. 9. Иллюстрация влияния толщины слоя сорбента на выходные кривые.

Кривая 1 соответствует $H=1$ мм, кривая 2 - $H=0.5$ мм, кривая $3-H=0.2$ мм, кривая 4 - $H=0.1$ мм.

Задача установки, изображенной на рис.1, состоит в деионозации порции раствора, выходящей в промежутке времени $0<t<T$, и восстановлении первоначального состояния сорбента. В модели содержится несколько параметров. Рассмотрим их влияние на процесс.

Чем выше приложенное напряжение $\Phi$, тем деионозация происходит эффективней, однако выбирать $\Phi$ выше 1.2 В нельзя, иначе, как известно, начинается гидролиз воды.

Вид кривой $C_{\text {out }}(t)$ зависит от соотношения между характерным временем поперечной диффузии в канале $t_{d i f}=\left(\frac{L}{\pi}\right)^{2} \frac{1}{D_{e x}}$, временем диффузии в поре $t_{\text {difpore }}=\left(\frac{H}{\pi}\right)^{2} \frac{1}{D_{i n}}$, временем прохождения раствора по каналу $t_{v}=\frac{L z}{v}$ и $T$. Если $t_{v}<T$, то при $t<t_{v}$ кривая $C_{\text {out }}(t)$ монотонно невозрастает, а при $t_{v}<t<T$ наблюдается ее рост. Если $t_{\text {difpore }}<t_{\text {dif }}<t_{v}<T$, то значение $C_{\min }$ определяется из условия баланса вещества при сорбции $L\left(C_{0}-C_{\min }\right)=2 \eta H\left(\psi\left(C_{\min }\right)-C_{0}\right)$, где функция $\psi$ связана с $I$ соотношением $\psi\left(\frac{c}{I(c)}\right)=c \cdot \psi(C)$ определяет концентрацию в порах, равновесную концентрации $C$ в канале.

Функции $I$ и $\psi$ растут с уменьшением $R_{0}$. Соответственно растет общее количество целевого компонента, которое может быть извлечено из раствора, при фиксированном $\eta$.

Если процесс будет построен циклическим образом, то максимальное количество электролита, отобранного из раствора за один цикл, составляет $Q=L z \cdot 2 H \eta\left(\psi\left(C_{0}\right)-C_{0}\right)$. Однако такое извлеченное $Q$ приходится на большое количество пропущенного раствора.

Пусть важно не количество извлеченного компонента, а количество выходящего раствора при условии на степень очистки: $C_{\text {out }}(t) \leq C_{c l}$, где $C_{\min }<C_{c l}<C_{0}$. Это 
условие будет выполнено на некотором промежутке времени $t_{1} \leq t \leq t_{2}$, где $C_{\text {out }}\left(t_{1,2}\right)=C_{c l}$. В случае $t_{\text {difpore }}<t_{d i f}<t_{v}<T$ можно приближенно определить $t_{1}$ и $t_{2}$ аналитически. Рассмотрим решение уравнения диффузии в направлении $x$ для канала. Удерживая главную моду при разложении решения уравнения диффузии по собственным функциям канала, находим: $C_{c l}-C_{\min }=\left(C_{0}-C_{\min }\right) e^{-\frac{\pi^{2} D}{L^{2}} t_{1}}$. Отсюда определяем $t_{1}=\frac{L^{2}}{\pi^{2} D} \ln \left(\frac{C_{0}-C_{\min }}{C_{c l}-C_{\min }}\right)$.

В рассматриваемом случае при $t \geq 2 t_{1}$ можем считать концентрацию вещества в канале не зависящей от $x$ и равновесной концентрации в порах. Условие сохранения вещества дает:

$\frac{\partial}{\partial t}(L C+2 \eta H \psi(C))+v L \frac{\partial C}{\partial z}=0$. Отсюда скорость переноса уровня $C_{c l}$ равна $v\left(1+2 \eta \frac{H}{L} \frac{\partial \psi\left(C_{c l}\right)}{\partial C}\right)^{-1}$ и $t_{2}=\frac{L z}{v_{c l}}$. В результате, количество раствора, удовлетворяющее условию $C_{\text {out }}(t) \leq C_{c l}$, будет максимальным при тех значениях $L$ и $v$, при которых максимальна величина

$$
L v\left(t_{2}-t_{1}\right)=L z\left(L+2 H \eta \frac{\partial \psi}{\partial C}\left(C_{c l}\right)\right)-\frac{L^{3} v}{\pi^{2} D} \ln \left(\frac{C_{0}-C_{\min }}{C_{c l}-C_{\min }}\right) .
$$

Для случая, когда нужно деионизировать наибольшее количество раствора при условии $\frac{1}{T} \int_{0}^{T} C_{\text {out }}(t) d t=C_{c l}$, получить аналитическое выражение, из которого можно определить оптимальные значения $L$ и $v$, не представляется возможным. Необходимо их подбирать, на основе численного моделирования решения задачи при различных значениях параметров.

\section{Учет конечной проводимости в модели}

Для учета конечной проводимости в описанную выше модель необходимо добавить уравнения, описывающие изменение плотности электрического заряда на поверхности пор аэрогеля как функции координаты х. При этом уравнения (8)-(12) остаются неизменными, а вместо уравнения (5) получаем систему (13)-(15)

$$
\begin{aligned}
& \frac{\partial^{2} U}{\partial x^{2}}= \frac{1}{S}\left(\left.R_{0} \cdot \frac{\partial u}{\partial r}\right|_{r=R_{0}}-\frac{q}{\varepsilon \varepsilon_{0} k T} Q\right) \text { с условиями: } \\
&\left.\frac{\partial U}{\partial x}\right|_{x=0}=2 \mu \sqrt{C_{x=0}} \operatorname{sh} \frac{U}{2},\left.U\right|_{x=H}=\frac{q}{k T} \frac{\Phi}{2} \\
& \frac{\partial Q}{\partial t}= \frac{\lambda}{\varepsilon \varepsilon_{0}}(1-\eta)\left(\left.\frac{\varepsilon \varepsilon_{0} k T}{q} R_{0} \cdot \frac{\partial u}{\partial r}\right|_{r=R_{0}}-Q\right) \text { с условием }\left.Q\right|_{t=0}=0 \\
& \frac{1}{r} \frac{\partial}{\partial r}\left(r \frac{\partial u}{\partial r}\right)=\mu \cdot \frac{\bar{C}}{\frac{1}{R_{0}{ }^{2}} \cdot \int_{0}^{R_{0}}\left(e^{u}+e^{-u}\right) \cdot r d r} \cdot\left(e^{u}-e^{-u}\right) \cdot \theta \\
& \text { С условиями: }\left.\frac{\partial u}{\partial r}\right|_{r=0}=0,\left.u\right|_{r=R_{0}}=U
\end{aligned}
$$

Здесь $\eta$ - порозность аэрогеля, $S$ - поперечная площадь аэрогеля, $U$ - электрический потенциал на поверхности пор аэрогеля, $q$ - заряд электрона, $Q$ - погон- 
ная плотность заряда внутри нитей аэрогеля, $\sigma$ - плотность заряда на концах нитей аэрогеля, $\lambda$ - удельная проводимость [Cм/м].

На рис.10 показаны результаты численных расчетов с помощью приведенной модели. Значения всех параметров соответствуют указанным по умолчанию перед рис. 4 кроме $H=1$ мм.

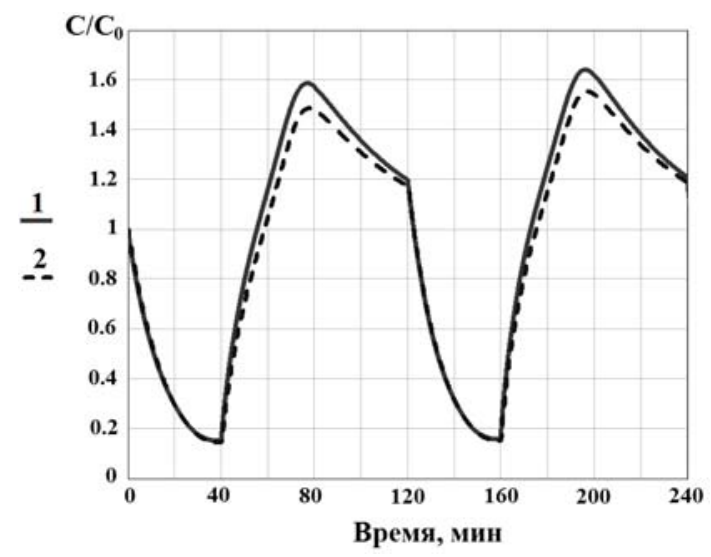

Рис. 10. Выходные кривые, полученные при моделировании циклического режима работы аэрогелей различной электрической проводимости.

Кривая 1 соответствует $\lambda=2000 \frac{C M}{M}$, кривая 2 соответствует $\lambda=10^{-3} \frac{C M}{M}$.

Как видно из рис. 10, электропроводимость аэрогеля может меняться в широких пределах, при этом выходные кривые слабо отличаются друг от друга.

\section{Заключение}

В работе исследованы различные математические модели процесса деионизации растворов с помощью сорбции на аэрогельных электродах и построен алгоритм количественного расчета условий равновесия, переноса и диффузии ионов. Приведены примеры расчета процесса деионизации в циклическом режиме.

Возникновение разности потенциалов между раствором в канале и порах аэрогельных электродов приводит к сдвигу равновесия концентраций ионов в соответствие с законом Больцмана. Это определяет втягивание ионов в сорбент, создание распределенного слоя ионов у поверхности, а при мелких порах и низких концентрациях раствора, кроме того, и распределенного заряда во всем объеме пор.

\section{Список литературы}

1. Al Marzooqi F.A., Al Ghaferi A.A., Saadat I., Hilal N. // Desalination. 2014. Vol. 342. pp. 3-15.

2. Zhao R.; Biesheuvel P.M.; van der Wal A. // Energy \& Environmental Science. 2012. Vol. 5. No 11. pp. 9520-9527. doi:10.1039/c2ee21737f.

3. Kim T., Dykstra J.E., Porada S, van der Wal A. et al. // J. Colloid Interface Science. 2014. Vol. 446. pp. 317-326. doi:10.1016/j.jcis.2014.08.041.
4. Suss M.E., Porada S., Sun X., Biesheuvel P.M. et al. // Energy \& Environmental Science. 2015. Vol .8. No 8.p p. 2296-2319. doi:10.1039/C5EE00519A.

5. Porada S., Zhao R., van der Wal A., Presser V. et al. // Progress in Materials Science. 2013. Vol. 58. No 8. pp. 1388-1442. doi:10.1016/j.pmatsci.2013.03.005.

6. Anderson M.A., Cudero A.L., Palma J. // Electrochimica Acta. 2010. Vol. 55. No 12. pp. 3845-3856. doi:10.1016/j.electacta.2010.02.012. 
7. Вольфкович Ю.М., Михалин А.А., Бограчев Д.А., Сосенкин В.Е. // Электрохимия. 2012. Т. 48. № 4. С. 467-477.

8. Oren Y. // Desalination. 2008. Vol. 228. pp.10-29.

9. Rasines G., Lavela P., Macías C., Haro M. et al. // J. Electroanalytical Chem. 2012. Vol. 671. pp. 92-98.

10. Marmanis D., Christoforidis A., Ouzounis K., Dermentzis K. // Global NEST Journal. 2014. Vol. 16. No 4. pp. 609-615.

11. Iozzo D.A.B., Tong M., Wu G., Furlani E.P. // J. Phys. Chem. C. 2015. Vol. 119. No 45. pp. 25235-25242.

12. Kirby B.J. The diffuse structure of the electrical double layer // www.kirbyresearch.com/index.cfm/wrap/textbo ok/microfluidicsnanofluidics.html

13. Yang K., Ying T., Yiacoumi S., Tsouris C. et al. // Langmuir. 2001. Vol.17. pp. 19611969.

\section{References}

1. Al Marzooqi F.A., Al Ghaferi A.A., Saadat I., Hilal N., Desalination, 2014, Vol. 342, pp. 3-15.

2. Zhao R.; Biesheuvel P.M.; van der Wal A., Energy \& Environmental Science, 2012, Vol. 5, No 11, pp. 9520-9527. doi:10.1039/c2ee21737f.

3. Kim T., Dykstra J.E., Porada S, van der Wal A. et al., J. Colloid Interface Science, 2014, Vol. 446, pp. 317-326. doi:10.1016/j.jcis.2014.08.041.

4. Suss M.E., Porada S., Sun X., Biesheuvel P.M. et al., Energy \& Environmental Science. 2015, Vol. 8, No 8, pp. 2296-2319. doi:10.1039/C5EE00519A.

5. Porada S., Zhao R., van der Wal A., Presser V. et al., Progress in Materials Science, 2013, Vol. 58, No 8, pp. 1388-1442. doi:10.1016/j.pmatsci.2013.03.005.

6. Anderson M.A., Cudero A.L., Palma J., Electrochimica Acta, 2010, Vol. 55, No 12, pp. 3845-3856. doi:10.1016/j.electacta.2010.02.012.

7. Vol'fkovich Yu.M., Mikhalin A.A., Bograchev D.A., Sosenkin V.E., Elektrokhimiya, 2012, Vol. 48, No 4, pp. 467-477.

8. Oren Y., Desalination, 2008, Vol. 228, pp.10-29.
14. Rios Perez C.A., Demirer O.N., Clifton, R.L., Naylor R.M. et al. // J. Electrochem. Soc. 2013. Vol. 160. No 3. pp. E13-E21.

15. Robinson D.B., Wu Ch. M., Jacobs B.W. // J. Electrochem. Soc. 2010. Vol. 157. No 8. pp. A912-A918.

16. Honda K., Yoshimura M., Kawakita K., Fujishima A. et al. // J. Electrochem. Soc. 2004. Vol. 151. No 4. pp. A532-A541.

17. Hoyt N.C., Wainright J.W., Savinell R.F. // J. Electrochem. Soc. 2015. Vol. 162. No 4. pp. A652-A657.

18. Niu C., Sichel E.K., Hoch R., Moy D. et al. // Appl. Phys. Lett. 1997. Vol. 70. No 11. pp.1480-1482.

19. Hemmatifar A., Stadermann M., Santiago J.G. // J. Phys. Chem. C. 2015. Vol. 119. No 44. pp. 24681-24694.

20. Gaberlich C.J., Xu P., Cohen Y. // Sustainability Science and Engineering. 2010. Vol. 2. pp. 295-326.

21. Suss M.E. // J. Electrochem. Soc. 2017. Vol. 164. No 9. pp. E270-E275.

9. Rasines G., Lavela P., Macías C., Haro M. et al., J. Electroanalytical Chem, 2012, Vol. 671, pp. 92-98.

10. Marmanis D., Christoforidis A., Ouzounis K., Dermentzis K., Global NEST Journal, 2014, Vol. 16, No 4, pp. 609-615.

11. Iozzo D.A.B., Tong M., Wu G., Furlani E.P., J. Phys. Chem. C, 2015, Vol. 119, No 45, pp. 25235-25242.

12. Kirby B.J. The diffuse structure of the electrical double layer, www.kirbyresearch.com/index.cfm/wrap/textbo ok/microfluidicsnanofluidics.html

13. Yang K., Ying T., Yiacoumi S., Tsouris C. et al., Langmuir, 2001, Vol. 17, pp. 19611969.

14. Rios Perez C.A., Demirer O.N., Clifton, R.L., Naylor R.M. et al., J. Electrochem. Soc., 2013, Vol. 160, No 3, pp. E13-E21.

15. Robinson D.B., Wu Ch. M., Jacobs B.W., J. Electrochem. Soc. 2010, Vol. 157, No 8, pp. A912-A918.

16. Honda K., Yoshimura M., Kawakita K., Fujishima A. et al., J. Electrochem. Soc., 2004, Vol. 151, No 4, pp. A532-A541.

17. Hoyt N.C., Wainright J.W., Savinell R.F., J. Electrochem. Soc., 2015, Vol. 162, No 4, pp. A652-A657. 
18. Niu C., Sichel E.K., Hoch R., Moy D. et al. , Appl. Phys. Lett. 1997. Vol. 70. No 11. pp. 1480-1482.

19. Hemmatifar A., Stadermann M., Santiago J.G., J. Phys. Chem. C, 2015, Vol. 119, No 44, pp. 24681-24694.

Тихонов Николай Андреевич - д.ф.м.н., профессор кафедры математики физического факультета МГУ им. М.В. Ломоносова, Москва

Токмачев Михаил Геннадьевич - к.ф.м.н., старший преподаватель кафедры математики физического факультета МГУ им. М.В. Ломоносова, Москва
20. Gaberlich C.J., Xu P., Cohen Y., Sustainability Science and Engineering, 2010, Vol. 2, pp. 295-326.

21. Suss M.E., J. Electrochem. Soc., 2017, Vol. 164, No 9, pp. E270-E275.

Tikhonov Nikolai A. - Grand PhD in Physicomathematical sciences, Professor of the mathematical department of physical faculty, Moscow Lomonosov State University, Moscow

Tokmachev Mikhail G. - PhD in Physicomathematical sciences, senior lecturer of the mathematical department of physical faculty, Moscow Lomonosov State University, Moscow Email: miket@mail.ru 